\title{
Multilayer Modal Actuator-Based Piezoelectric Transformers
}

\author{
Yao-Tien Huang, Wen-Jong Wu, Yen-Chieh Wang, and Chih-Kung Lee
}

\begin{abstract}
An innovative, multilayer piezoelectric transformer equipped with a full modal filtering input electrode is reported herein. This modal-shaped electrode, based on the orthogonal property of structural vibration modes, is characterized by full modal filtering to ensure that only the desired vibration mode is excited during operation. The newly developed piezoelectric transformer is comprised of three layers: a multilayered input layer, an insulation layer, and a single output layer. The electrode shape of the input layer is derived from its structural vibration modal shape, which takes advantage of the orthogonal property of the vibration modes to achieve a full modal filtering effect. The insulation layer possesses two functions: first, to couple the mechanical vibration energy between the input and output, and second, to provide electrical insulation between the two layers. To meet the two functions, a low temperature, cofired ceramic (LTCC) was used to provide the high mechanical rigidity and high electrical insulation. It can be shown that this newly developed piezoelectric transformer has the advantage of possessing a more efficient energy transfer and a wider optimal working frequency range when compared to traditional piezoelectric transformers. A multilayer piezoelectric, transformer-based inverter applicable for use in LCD monitors or portable displays is presented as well.
\end{abstract}

\section{INTRODUCTION}

$\mathrm{T}$ HE advantages of using piezoelectric transformers to light LCD monitor backlights include its thin thickness, a small temperature rise, and a low electromagnetic interference (EMI) noise. In addition, a piezoelectric transformer is more suitable for mass production than traditional, coil-based transformers. If piezoelectric transformers are used to light cold cathode fluorescent lamps (CCFLs) in today's large-size LCD monitors, an extra high stepup ratio capability is required. To achieve such a high stepup ratio, a multilayer, piezoelectric transformer [1]-[3] is typically adopted.

Adopting a modal actuator and a modal sensor design, the input electrode of a piezoelectric transformer was first reported by Lee in 1987 [4]-[6]. In these works, a quasimodal-shaped input electrode was incorporated in a Rosen-type piezoelectric transformer. Although the input distributed electrode did not represent the full modal strain due to the fact that the input electrode covered only

Manuscript received June 20, 2006; accepted September 14, 2006. The present study was financially supported from the National Science Council of Taiwan, through Grant NSC 95-2622-E-002-003.

Y.-T. Huang and C.-K. Lee are with the Institute of Applied Mechanics, National Taiwan University, Taipei, Taiwan (e-mail: cklee@ntu.edu.tw).

W.-J. Wu, Y.-C. Wang, and also C.-K. Lee are with the Department of Engineering Science and Ocean Engineering, National Taiwan University, Taipei, Taiwan.

Digital Object Identifier 10.1109/TUFFC.2007.249 half of the full piezoelectric transformer, the effect of the modal filtering with its roots based on the orthogonality condition of different modes was significant. The previously reported experimental results indicated that quasimodal, Rosen-type piezoelectric transformers can possess an output waveform of a lower, high-order harmonics when compared to traditional Rosen-type piezoelectric transformers, and that translate into a possible higher total energy transfer efficiency [7], [8]. Although modal sensor and actuator concepts can be adopted, some deficiencies still remain as the geometry constraint of a halflength actuator (input side of the transformer) prevents a modal actuator from completely eliminating the influences of the unwanted modes. In order to remove the abovementioned deficiencies and to further reduce the influence of the load impedance connected to the sensor part (output side) of piezoelectric transformers, a newly developed, full-modal piezoelectric transformer that incorporates an insulation layer to separate the sensor and the actuator layer is presented herein. The main idea behind developing such a modal electrode was to design the shape of a distributed electrode to match the surface stress distribution of the piezoelectric transformer. Our full-modal, piezoelectric transformer is different from the previously developed quasimodal piezoelectric transformer as, theoretically, our new, full-modal type can provide full modal filtering so as to prevent high-frequency noise from feeding into the piezoelectric transformer. It can be shown that our new, full-modal, piezoelectric transformer possesses a higher energy transfer efficiency and a larger optimal working frequency range when compared to previous piezoelectric transformers.

In order to provide the extra high stepup ratio for large-size LCD monitors, our newly developed piezoelectric transformer consists of a multilayer structure at the input layer [9], [10]. An innovative piezoelectric transformerbased inverter - which switches from a frequency-swept to a constant, current feedback control when the CCFL is turned on-was developed to further expand the advantages of this line of piezoelectric technology. In fact, a fullmodal, piezoelectric transformer that possesses higher energy transfer efficiency makes it not only easier for control, but it also ensures the presence of a higher stepup ratio and a lower temperature rise.

\section{Structure of Full-Modal Piezoelectric TRANSFORMER}

Our $26 \mathrm{~mm} \times 5 \mathrm{~mm} \times 5 \mathrm{~mm}$ piezoelectric transformer was manufactured by Eleceram Technology Co. 


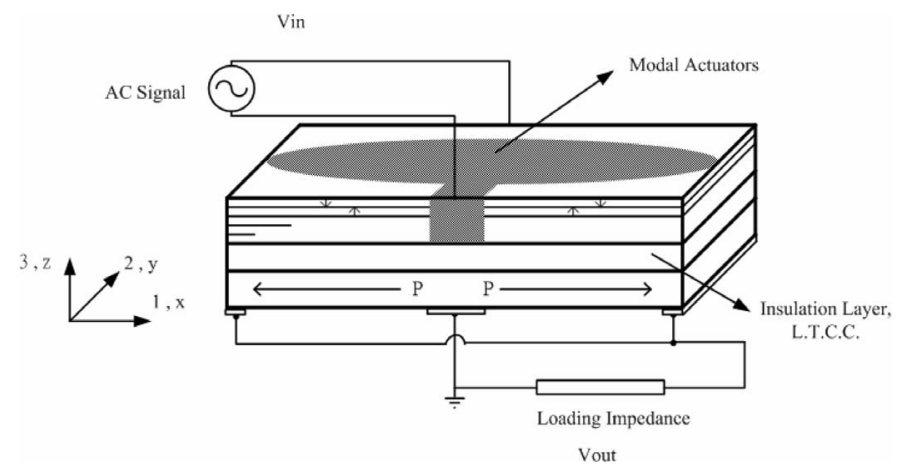

(a)

The odd-layer piezoelectric lamina

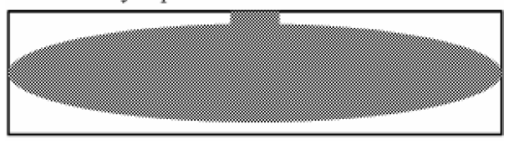

The even-layer piezoelectric lamina

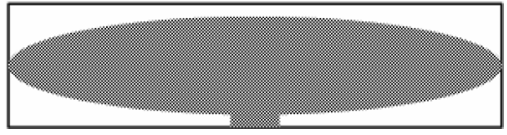

(b)

The odd-layer piezoelectric lamina

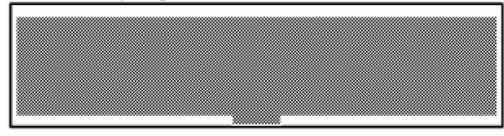

The even-layer piezoelectric lamina

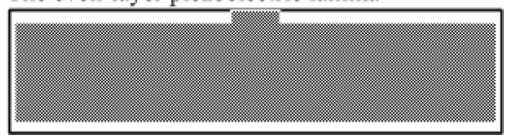

(c)

Fig. 1. (a) Schematic of a multilayer, full-modal, actuator-based piezoelectric transformer. (b) Input electrode of a full-modal, piezoelectric transformer. (c) Input electrode of an uniform electrode piezoelectric transformer. (Note: shaded area represents the surface electrode.)

Ltd., Taoyuan, Taiwan. We glued the middle insulation layer to the input and output terminals (Fig. 1). The multilayered input section with each layer poled and electrically connected in a parallel configuration created a surface electrode that was proportional to the modal strain of the desired operating mode. In Figs. 1(b) and (c), we can see the shape of the input electrode at which the odd-layer, piezoelectric lamina was connected to the left side of the piezoelectric transformer, and the even-layer connected to the right side of the piezoelectric transformer.

We knew that, in choosing a suitable insulation layer, we needed to consider the requirement for a high dielectric constant to insulate the output high voltage, and that it must be of high rigidity in order to transfer the vibration energy between the input and output. Thus, we chose a low-temperature, co-fired ceramic (LTCC) as the middle insulation layer. The single layer output section was poled opposite along the horizontal direction (the 1-direction), the central electrode was used as the output ground ter- minal, and the terminals at the two ends were connected together to drive the loads. Following traditional piezoelectric sign convention, the poling direction of each lamina within a piezoelectric laminate was denoted as a $3^{\prime}$ axis. That is, the electric output of the piezoelectric transformer was extracted from this axis, e.g., using $D_{3^{\prime}}^{\text {out }}$ as the output electric displacement.

The modal actuator layer had its poling direction in the 3-direction, and the externally applied electric field along the 3 -direction drove the entire piezoelectric transformer. The governing equation of a full-modal, piezoelectric transformer derived from the dynamics equation along the x-direction, thus can be represented by (1) [7], [8]:

$$
c \frac{\partial^{2} u(x, t)}{\partial x^{2}}-e_{31} m E_{3}^{\text {in }} \frac{\partial S(x)}{\partial x}=\rho \frac{\partial^{2} u(x, t)}{\partial t^{2}} .
$$

The second term represents the external forcing term, which is the coupling between the input electrical field along the 3 -direction $E_{3}^{\text {in }}$ and the piezoelectricity of the input section. In addition, $e_{31}$ is the piezoelectric constant; $S(x)$ represents the shape function of the input electrode along the 1-direction, $m$ is the number of the piezoelectric layers in the input section, $c$ is the effective elastic stiffness of the full-modal, piezoelectric transformer along the 1direction, and $u(x, t)$ represents the displacement along the 1-direction. In (1), the boundary conditions of this fullmodal, piezoelectric transformer can be represented by (2) and (3):

$$
\begin{aligned}
0 & =\left.\left.H W\left[c \frac{\partial u}{\partial x}-h_{3^{\prime} 3^{\prime}} D_{3^{\prime}}^{\text {out }}(x, t)\right]\right|_{x=0} \Rightarrow \frac{\partial u}{\partial x}\right|_{x=0} \\
& =\frac{h_{3^{\prime} 3^{\prime}} D_{3^{\prime}}^{\text {out }}(0, t)}{c}
\end{aligned}
$$

and

$$
\begin{aligned}
0 & =\left.\left.H W\left[c \frac{\partial u}{\partial x}-h_{3^{\prime} 3^{\prime}} D_{3^{\prime}}^{\text {out }}(x, t)\right]\right|_{x=l} \Rightarrow \frac{\partial u}{\partial x}\right|_{x=l} \\
& =\frac{h_{3^{\prime} 3^{\prime}} D_{3^{\prime}}^{\text {out }}(l, t)}{c}
\end{aligned}
$$

where $l$ is the length of the piezoelectric transformer, $D_{3^{\prime}}^{\text {out }}$ represents the output electric displacement at the two different ends $(x=0$ and $x=l)$ of the piezoelectric transformer. In (2) and (3), $h_{3^{\prime} 3^{\prime}}$ represents the stress/electric displacement constant of the output section, and $H$ and $W$ represent the thickness and width of the full-modal, piezoelectric transformer, respectively. The displacement field $u(x, t)$ along the 1-direction can be assumed to be:

$$
u(x, t)=\sum_{i=1}^{\infty} A_{i}(t) \varphi_{i}(x),
$$

where $\varphi_{i}(x)$ represents the modal coordinates, and $A_{i}(t)$ represents the magnitude along the modal coordinate. The modal coordinates can be shown as:

$$
\varphi_{i}=\sqrt{\frac{2}{l}} \cos \sqrt{\lambda_{i}} x \quad \lambda_{i}=\left(\frac{i \pi}{l}\right)^{2},
$$


where $i$ represents the $i$-th resonance mode. Substituting the displacement field into the governing equation, then performing an eigen-function expansion to the governing equation reduces the partial differential equation (Fig. 1) into an infinite set of ordinary differential equations:

$$
\begin{gathered}
\frac{d^{2} A_{i}(t)}{d t^{2}}+\frac{c \lambda_{i}}{\rho} A_{i}(t)= \\
q_{i}(t)+\sqrt{\frac{2}{l}}\left[(-1)^{i+1} \frac{h_{3^{\prime} 3^{\prime}} D_{3^{\prime}}^{\text {out }}(l, t)}{c}+\frac{h_{3^{\prime} 3^{\prime}} D_{3^{\prime}}^{\text {out }}(0, t)}{c}\right] \\
q_{i}(t)=-\frac{e_{31} m E_{3}^{\text {in }}}{\rho} \int_{0}^{l}\left(\frac{\partial S(x)}{\partial x}\right) \phi_{i}(x) d x
\end{gathered}
$$

The right side of (6) represents the external forcing term of the governing equation and the input electric field can be shown as $E_{3}^{\text {in }}$. In this paper, the electrode shape of the input section $S(x)$ possesses two forms: one takes a constant form of traditional piezoelectric transformers, and the other is proportional to $\partial \varphi_{i}(x) / \partial x$, i.e., the modal strain that corresponds to the mode of interest, to arrive at a full-modal actuator design. In (6), the last two forcing terms are contributed by the boundary conditions and represent electric displacement at the structural ends of the piezoelectric transformer. In our paper, we use a fullmodal, piezoelectric transformer to light a single CCFL, which was driven by connecting the two output electrodes together. With the symmetry of the two terminal ends and the opposite poling, the two terminals will have the same load impedance. Furthermore, if the piezoelectric transformer works in an open condition or is connected to a load of equal impedance at the two ends, the influences of the boundary conditions will cancel each other out for the case in which there is an odd mode resonance. Thus, the input electric field can be shown to be periodic, i.e.:

$$
E_{3}^{\text {in }}(t)=\left|E_{3}^{\text {in }}\right| \cos \omega t
$$

By combining the above, the governing equation can be rewritten as:

$$
\begin{aligned}
& \frac{d^{2} A_{i}(t)}{d t^{2}}+\frac{c \lambda_{i}}{\rho} A_{i}(t)=r_{i} \cos \omega t, \\
& r_{i}=-\frac{e_{31}\left|E_{3}^{\text {in }}\right|}{\rho} \sqrt{\frac{2}{l}}\left[\int_{0}^{l} \frac{\partial S(x)}{\partial x} \cos \left(\frac{i \pi x}{l}\right) d x\right],
\end{aligned}
$$

where $r_{i}$ is defined as the driving factor and is independent of time. The solution of (8) thus becomes:

$$
A_{i}(t)=\frac{r_{i} \cos \omega t}{\left(\omega_{i}^{2}-\omega^{2}\right)}, \quad \omega_{i}=\sqrt{\frac{c \lambda_{i}}{\rho}} .
$$

From (4) and (9), the displacement of the full-modal, piezoelectric transformer along the 1-direction can be represented as:

$$
\begin{aligned}
u(x, t)= & \sum_{i=1}^{\infty} \frac{r_{i} \cos \omega t}{\left(\omega_{i}^{2}-\omega^{2}\right)} \varphi_{i}(x) \\
= & \frac{r_{1} \cos \omega t}{\left(\omega_{1}^{2}-\omega^{2}\right)} \varphi_{1}(x)+\frac{r_{2} \cos \omega t}{\left(\omega_{2}^{2}-\omega^{2}\right)} \varphi_{2}(x) \\
& +\frac{r_{3} \cos \omega t}{\left(\omega_{3}^{2}-\omega^{2}\right)} \varphi_{3}(x)+\cdots+\frac{r_{n} \cos \omega t}{\left(\omega_{n}^{2}-\omega^{2}\right)} \varphi_{n}(x), \\
n= & \infty .
\end{aligned}
$$

The symbol $\omega$ represents the angular frequency of the input electric field, and $\omega_{i}$ is the resonant frequency of the $i$-th piezoelectric transformer vibration mode. From (10) we know that, when an input electric field possessing a single frequency is near the first resonant frequency, some energy will leak to other resonant modes if the driving factor $r_{i}$ is not equal to zero at other resonant frequencies. From this theoretical standpoint, we can see that the shape of the input electrode $S(x)$ may influence the distribution of the input energy, even if the input voltage is a single frequency. In order to verify the effect of the full modal electrode, a normalized driving factor $r_{i b}$ can be defined as:

$$
r_{i b}=-\frac{1}{i^{2}} \frac{r_{i}}{\frac{e_{31}\left|E_{3}^{\text {in }}\right|}{\rho} \sqrt{\frac{2}{l}}},
$$

In (11), the normalized driving factor was divided by $i^{2}$ and was used to represent displacement that is inversely proportional to $\omega^{2}$ in (10). The normalized driving factor in the paper then can be used to display the distribution of input energy when the shape of the input electrode is changed. In our paper, the multilayer piezoelectric transformer consists of two different kinds of input electrodes, one of which is a full-modal electrode, and the other is a traditional distributed type [see (12) and (13)]. More specifically,

$$
\begin{gathered}
S_{m}(x)=\sin \left(\frac{\pi x}{l}\right), \\
S_{d}(x)=H\left[x-(1-a) \frac{l}{2}\right]-H\left[x-(1+a) \frac{l}{2}\right],
\end{gathered}
$$

where $S_{m}(x)$ represents a full-modal electrode and $S_{d}(x)$ represents a traditional, uniform electrode. Substituting (12) and (13) into (11) yields the relationship between the resonant mode and the normalized driving factor (Fig. 2). In Fig. $2, r_{i b}$ (modal) and $r_{i b}$ (uniform) represent the normalized driving factors for a piezoelectric transformer of a full-modal electrode and a piezoelectric transformer of a distributed electrode, respectively.

It is clear from Fig. 2 that the normalized driving factor for a full-modal piezoelectric transformer is zero, except at the first resonant frequency. More specifically, a full-modal electrode can provide the absolute band-pass filtering for the piezoelectric transformer. From Fig. 2, we see that, even if a single-frequency input voltage has its driving frequency located very close to the first resonant frequency, there still will be some energy that will be discharged to 


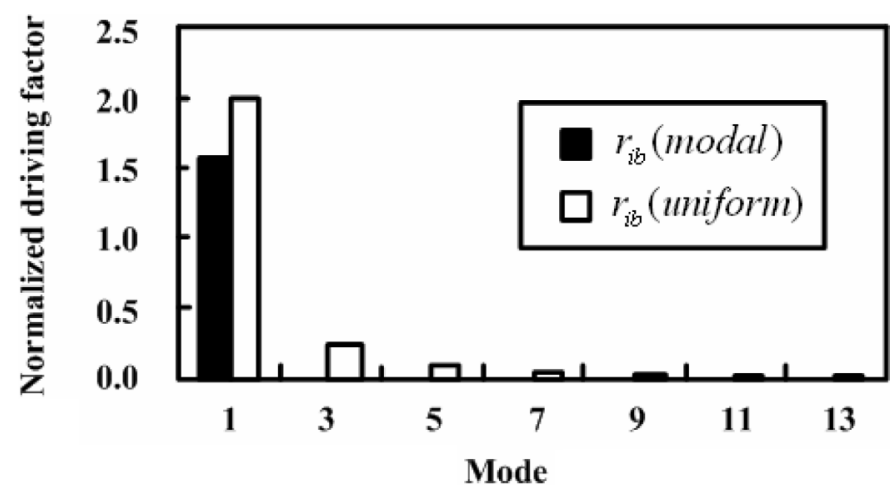

Fig. 2. Normalized driving factors for piezoelectric transformers with full-modal electrodes and with distributed/uniform electrodes.

affect other resonant frequencies for a piezoelectric transformer possessing a uniform electrode. This discharged energy affects the piezoelectric transformer by lowering its energy transfer efficiency. However, a piezoelectric transformer with a full-modal electrode will possess full-modal filtering ability such that the input energy will not leak to other resonant modes. Thus, with no energy leakage, higher energy transfer efficiency can be obtained.

With an attempt to understand the input energy distribution more clearly, an impedance analyzer (4294, Agilent Technologies, Santa Clara, CA) was used to measure the input impedance of two piezoelectric transformers, one with full-modal electrodes and one with uniform distributed electrodes, when the output electrodes were shorted to the ground. Fig. 3 shows the input impedance of a piezoelectric transformer of a traditional uniform electrode. It is clear that, if the piezoelectric transformer is not operated near a structural resonant frequency, it possesses characteristics of a static capacitance such as that of a typical piezoelectric actuator. If the driving frequency is placed near a structural resonant frequency, the input impedance of a piezoelectric transformer has both an antiresonant peak and a resonant peak.

It can be seen from the experimental results that the first resonant frequency was about $68.8 \mathrm{kHz}$, which was set as the designed working frequency for the full-modal, piezoelectric transformer. At this first resonant frequency, the input impedance of the full-modal, piezoelectric transformer showed an antiresonant peak and a resonant peak similar to that of the uniform electrode piezoelectric transformer. The electric energy was delivered in full by the piezoelectric transformer at near the resonant peak. However, the input impedance of a full-modal, piezoelectric transformer is vastly different from that of a uniform distributed type. The input impedance of the full-modal, piezoelectric transformer at the third and the fifth resonant frequencies are similar to a static capacitance. In addition, the phase difference of the input impedance at those conditions is near minus 90 degrees, which means that the full-modal, piezoelectric transformer does not deliver any electric energy through the third and the fifth resonant modes. This experimental result matches well with the theoretical prediction.

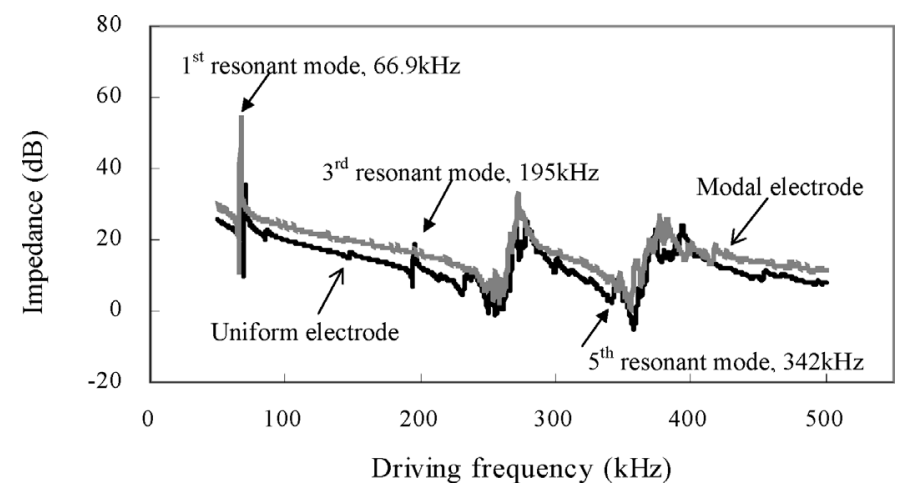

(a)

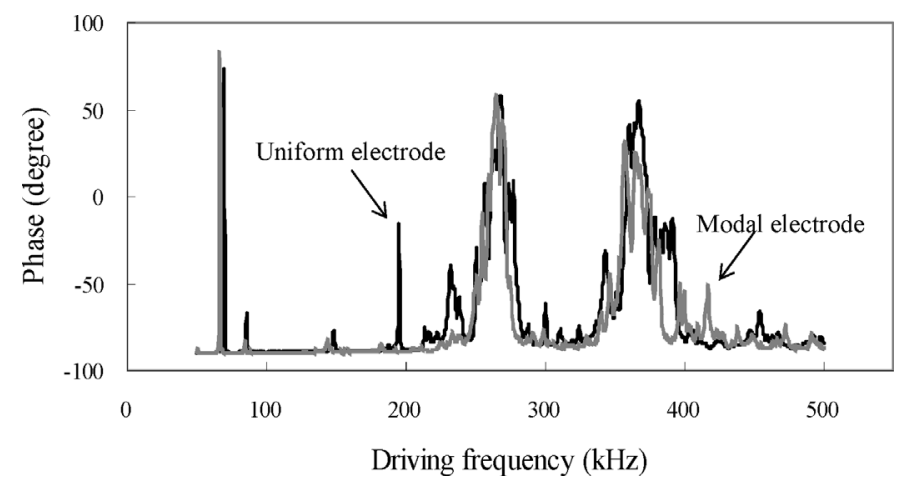

(b)

Fig. 3. Comparison of modal and uniform piezoelectric transformers. (a) Input impedance. (b) Phase.

In addition to the input impedance, the stepup ratios of the two different kinds of piezoelectric transformers also were measured using a network analyzer (4395A, Agilent Technologies) when the output electrode was connected to a $1 \mathrm{M} \Omega$ probe impedance. The measured stepup ratio and the phase angle are shown in Fig. 4. It can be seen from Fig. 4(a) that the gain of the full-modal, piezoelectric transformer at the first resonant frequency was 63.4, and 65.6 for a traditional uniform, electrode-based type. At the third resonant frequency, the stepup ratio of the traditional and the modal-based, piezoelectric transformer was 21.2 and 1.78, respectively. In other words, the gain was minimal at the third resonant frequency for the modalbased, piezoelectric transformer. The experimental result verifies the modal filtering effect and matches the theoretical prediction. In summary, the experimental results shown in Figs. 3 and 4 demonstrate the excellent modal filtering effect on the input voltage when a modal-based input electrode is adopted.

Traditionally, piezoelectric, transformer-based inverters use a MOSFET (metal-oxide semiconductor field effect transistors) to switch on an LC (inductor-capacitor) resonant tank. If the switching frequency coincides with the resonant frequency of the LC resonant tank, the driving voltage delivered to the piezoelectric transformer will be close to sinusoidal with the same frequency as the switching frequency. When an inductor is absent, and thus the resonant condition cannot be met, the switch of the MOSFET on the piezoelectric transformer delivers a square 

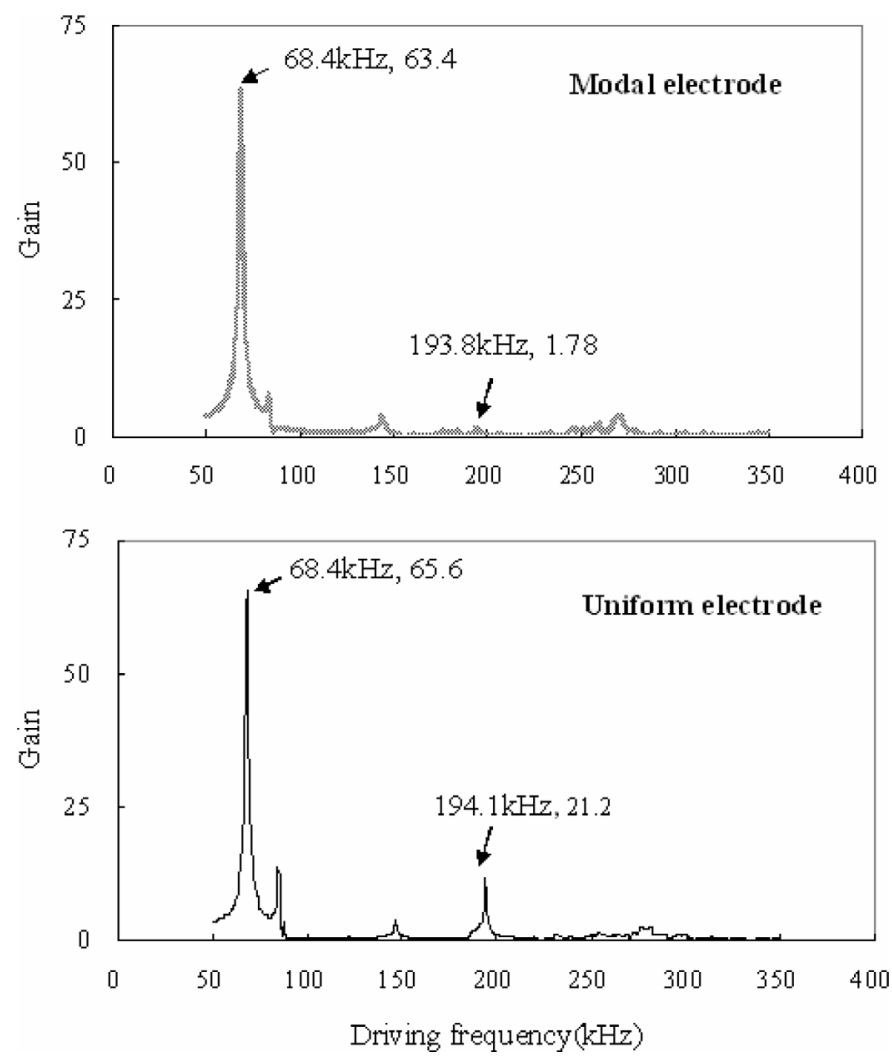

(a)

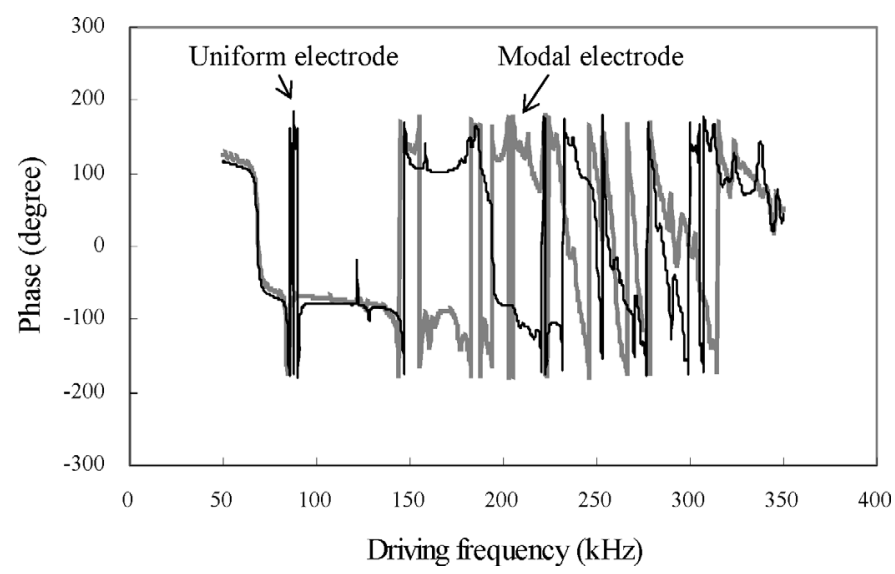

(b)

Fig. 4. Comparison of modal and uniform piezoelectric transformers. (a) Gain/stepup ratio. (b) Phase.

waveform to the piezoelectric transformer. The higher harmonics of the square waveform then will excite unwanted vibration modes such that the energy transfer efficiency will be degraded. Even though an inductor eliminates some of the higher harmonics of the driving waveform, the remaining higher harmonic noise cannot be further reduced to a lower desired level. A full modal piezoelectric transformer as presented above can provide the modal filtering effect by taking advantage of the orthogonality of the piezoelectric transformer structural mode. In other words, the newly developed, full-modal, piezoelectric transformer can prevent the input voltage noise from inducing undesirable, high-order vibration. In other words, an input elec- trode based on a modal actuator concept can be regarded as a band-pass filter that can clean up the noise embedded within an input voltage waveform.

\section{Operating a Full-Modal, Piezoelectric Transformer Under a High-Power Condition}

To determine an optimal working condition, factors such as driving frequency, stepup ratio, and efficiency must be examined. As the stepup ratio and driving frequency already have been evaluated above, we need to next consider efficiency, especially when operating under a high-power condition. A function generator (3314, Hewlett Packard, Palo Alto, CA) and a power amplifier (4052, NF Instruments, Yokohama, Japan) were used to provide the different frequency input voltages. 10X and 100X voltage probes (P6139A and P5100, Tektronix Inc., Beaverton, OR) were used to measure the input and output voltage waveforms. A current probe and a current amplifier (A6302 and AM503B, Tektronix Inc.) were used to measure the input current. The output current was obtained by measuring the voltage across a $100 \Omega$ resistor that was connected between the load and ground. The input voltage was found to be $6 \mathrm{Vrms}$, and the driving frequency was found to vary from $63 \mathrm{kHz}$ to $70 \mathrm{kHz}$ [11].

From Fig. 5(a), we can see that the stepup ratio for different load impedances lies between the ranges of 30 to 80. The energy transfer efficiency was found to be higher than $90 \%$ when the load impedance was between the ranges of $80 \mathrm{k} \Omega$ to $200 \mathrm{k} \Omega$. In addition, the optimal working frequency range was determined to be between $66 \mathrm{kHz}$ and $67.5 \mathrm{kHz}$. To simulate the load impedance effect of a $300-\mathrm{mm}$ CCFL, a $100 \mathrm{k} \Omega$ load was used and connected to the output electrode. A sinusoidal input voltage then was used to drive the full-modal, piezoelectric transformer, and the driving frequency was found to vary from $66 \mathrm{kHz}$ to $68.5 \mathrm{kHz}$. The input power was set at about $3.5 \mathrm{~W}$, and the energy transfer efficiencies of the two different types of piezoelectric transformers were measured (see Fig. 6). The experimental results clearly indicate that a piezoelectric transformer with a full-modal input electrode possesses higher total energy efficiency. The efficiency of the full-modal, piezoelectric transformer at $68.5 \mathrm{kHz}$ was $13.5 \%$ higher than that obtained from a traditional, uniform, input electrode piezoelectric transformer. Also, we can see that the optimal working frequency range of a full-modal, piezoelectric transformer, which possesses high energy transfer efficiency, is wider in range than that of a piezoelectric transformer with a uniform input electrode.

From previous experimental results, we know that the optimal operating region of a piezoelectric transformer is located somewhere between the resonant frequency and antiresonant frequency [12], [13]. In order to further verify the optimal working frequency range, the input impedances of the two piezoelectric transformers were measured. The load impedances at $100 \mathrm{k} \Omega$ and $200 \mathrm{k} \Omega$ were connected to the output section of the piezoelectric 


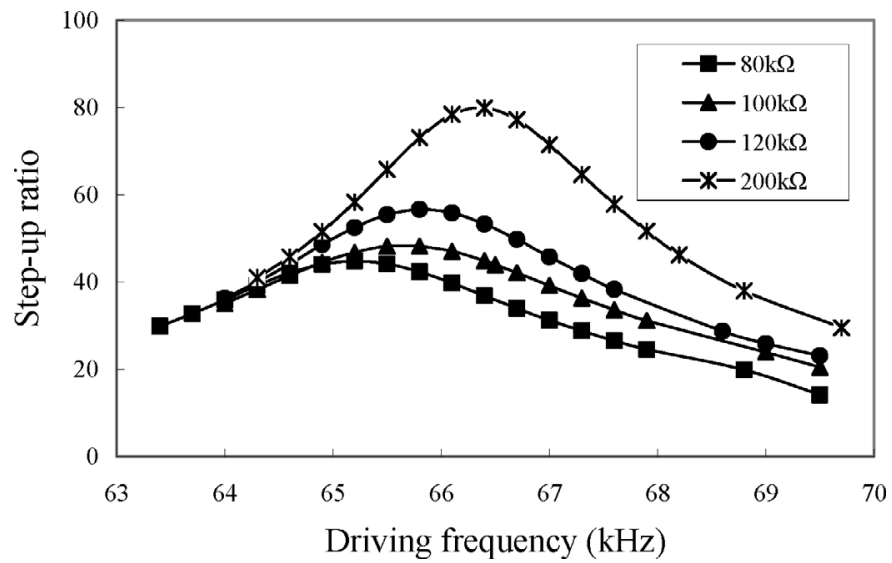

(a)

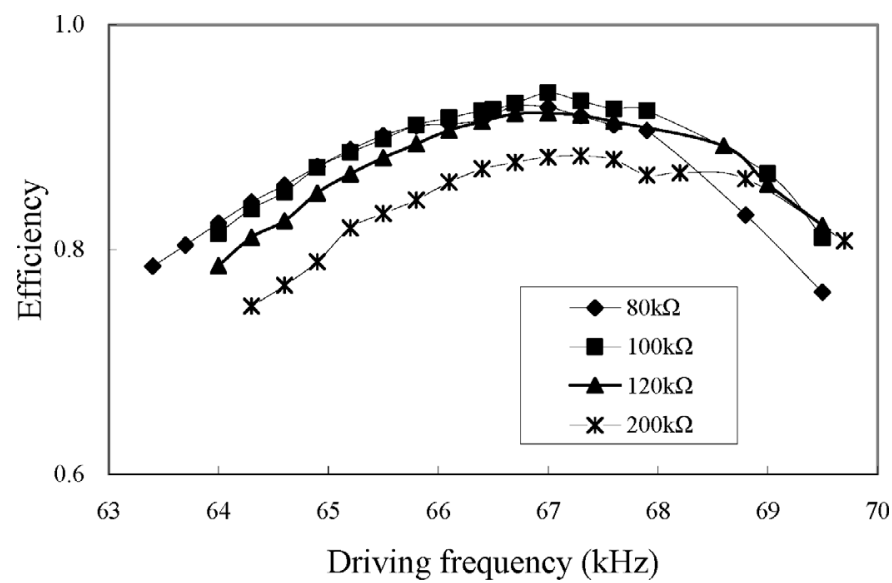

(b)

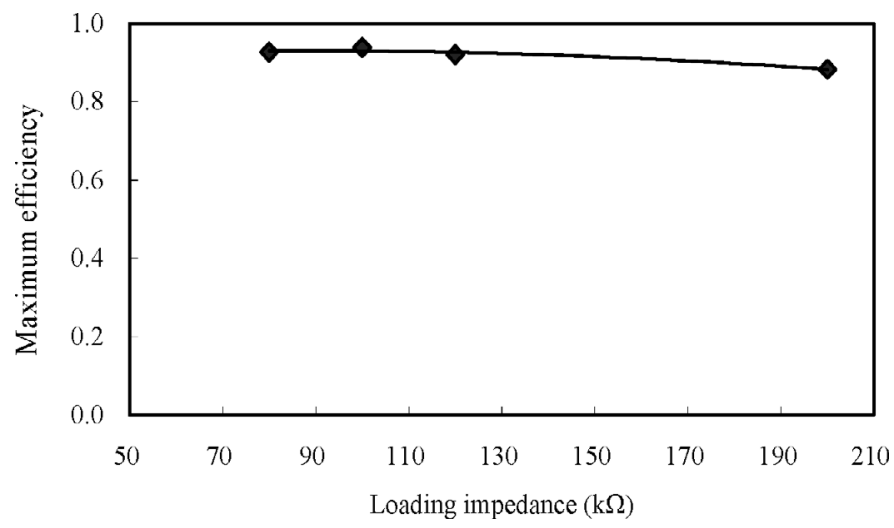

(c)

Fig. 5. Modal piezoelectric transfer under a high-power operating condition at different load impedances. (a) Stepup ratio. (b) Efficiency. (c) Maximum efficiency.

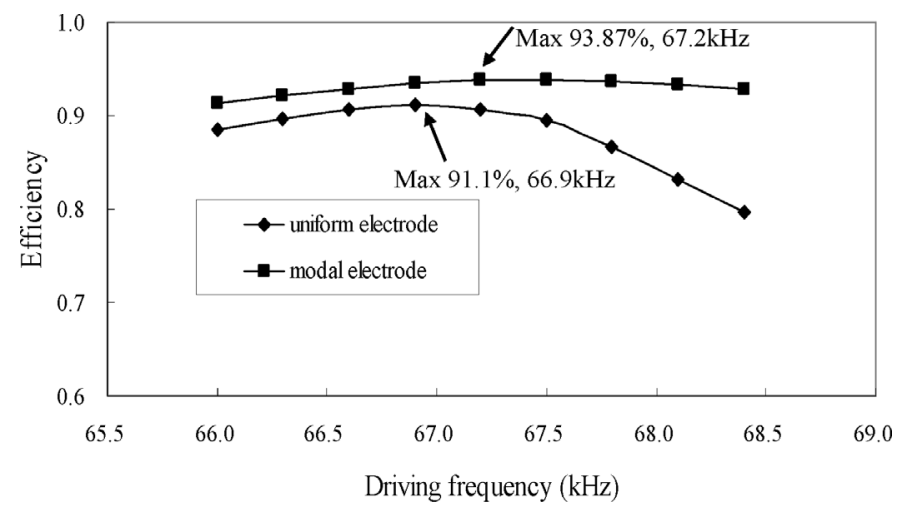

Fig. 6. Efficiency comparison for full-modal and uniform piezoelectric transformers.

transformer to simulate a CCFL load condition. The experimental results are shown in Fig. 7. Fig. 7(a) represents the magnitude of the input impedance of the two piezoelectric transformers, and Fig. 7(b) represents the phase angle of the input impedance. With an attempt to compare the optimal operating range between the resonant frequency and the antiresonant frequency, Fig. 8 was redrawn by normalizing Fig. 7. The minimum input impedances of the two different piezoelectric transformers in Fig. 7(a) were normalized to equal one, and the frequency at minimum impedance also was assumed to be equal to one. The maximum phase angle of the two different piezoelectric transformers shown in Fig. 7(b) were normalized to be equal to one, and the frequency for possessing maximum phase angle was normalized to be equal to one as well (Fig. 8). From Fig. 8, we can observe the corresponding phase angle change between the resonant and the antiresonant frequencies within the optimal operating range. It is quite clear from the data shown that a full-modal, piezoelectric transformer has more of a wider optimal frequency range when compared to a piezoelectric transformer with uniform electrodes. In summary, a full modal electrode possesses better total energy transfer efficiency.

\section{Properties of a 300 mm CCFL And Driving Circuit}

A piezoelectric, transformer-based inverter was developed to demonstrate the merits of the newly developed piezoelectric transformer. A 300-mm long, CCFL used in today's 14-inch notebook displays was used as the electric load. The properties of the 300-mm CCFL were measured (Fig. 9), and steps were taken to ensure that the load impedance matched well with the optimal load impedance for the piezoelectric transformer.

From Fig. 9, we can see that the CCFL impedance changed from $80 \mathrm{k} \Omega$ to $200 \mathrm{k} \Omega$ when power changed from $2 \mathrm{~W}$ to $4 \mathrm{~W}$. Comparing this result with the data shown in Fig. 5(c), we can see that the maximum energy transfer efficiency achieved was higher than $90 \%$. The driving circuit of the piezoelectric-based inverter can be seen in 


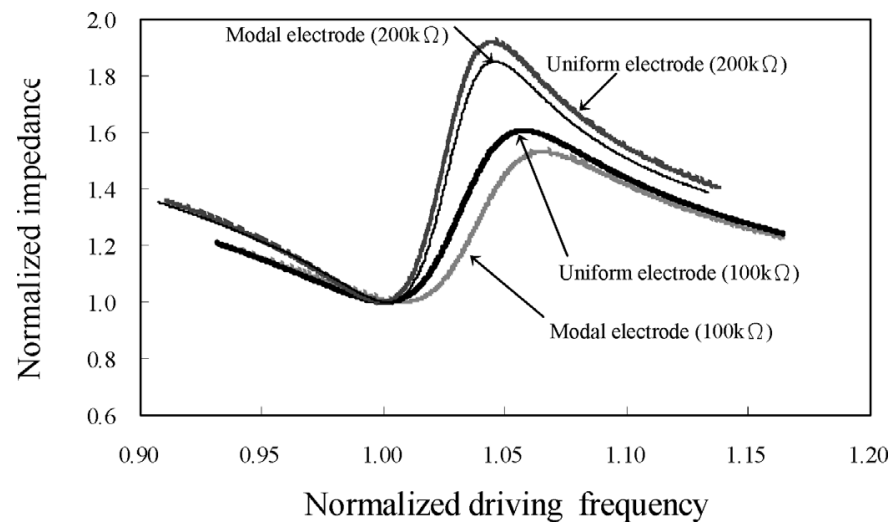

(a)

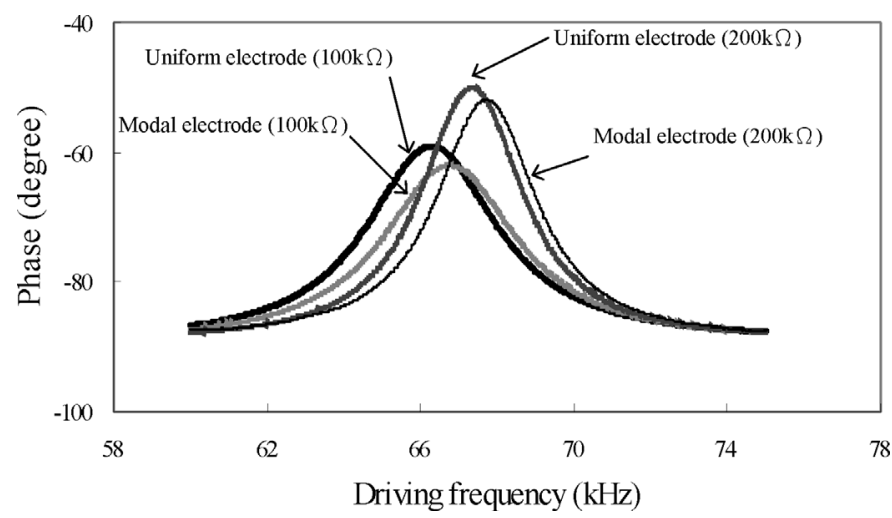

(b)

Fig. 7. Input impedance of modal and uniform piezoelectric transformers at $100 \mathrm{k} \Omega$ and $200 \mathrm{k} \Omega$ load. (a) Magnitude. (b) Phase.

Fig. 10. An UCC3976 IC (Texas Instruments, Dallas, TX) was used to sweep the frequency from high to low in order to light the CCFL. In addition, a PMOS IRF9Z24NS and NMOS IRF540S (International Rectifier Corporation, El Segundo, CA) were used to form a half-bridge driver to power the full-modal, piezoelectric transformer.

\section{Experimental Results of a Piezoelectric-Based InVERTER}

In order to verify the full-modal filtering effect, a driving circuit was used to drive the two different kinds of the piezoelectric transformer. The first type was a fullmodal, piezoelectric transformer, and the second type was a piezoelectric transformer with uniform electrodes. An inductor was placed in front of the piezoelectric transformer to produce the LC resonance in order to filter out the high-frequency noise embedded within the input voltage (Fig. 9). The static input capacitances of the two piezoelectric transformers will be different due to the shape differences of the input electrodes. In our experiments, the input capacitance of the full-modal, piezoelectric transformer was measured to be $90 \mathrm{nF}$, and $170 \mathrm{nF}$ was the measured input capacitance for the piezoelectric transformer with uniform electrodes. As it was not possible to locate a single inductor to perfectly match both piezo-

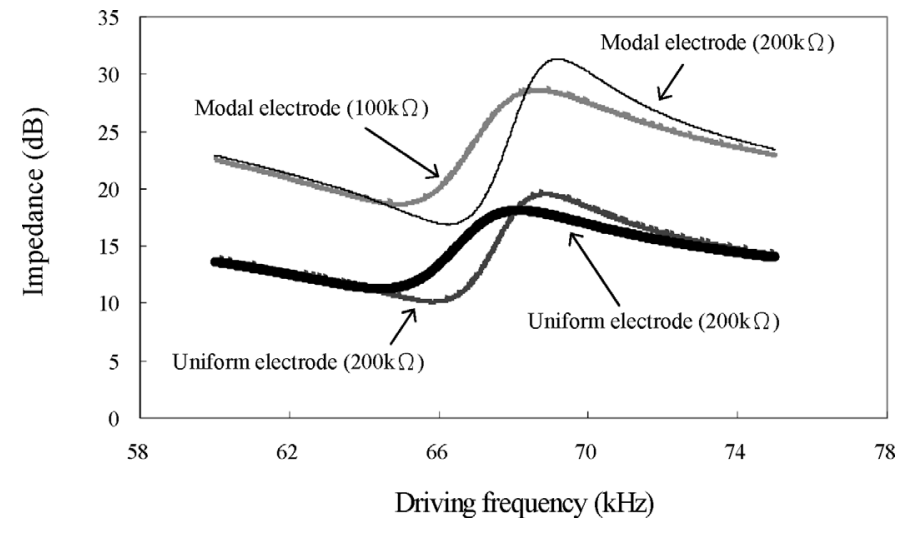

(a)

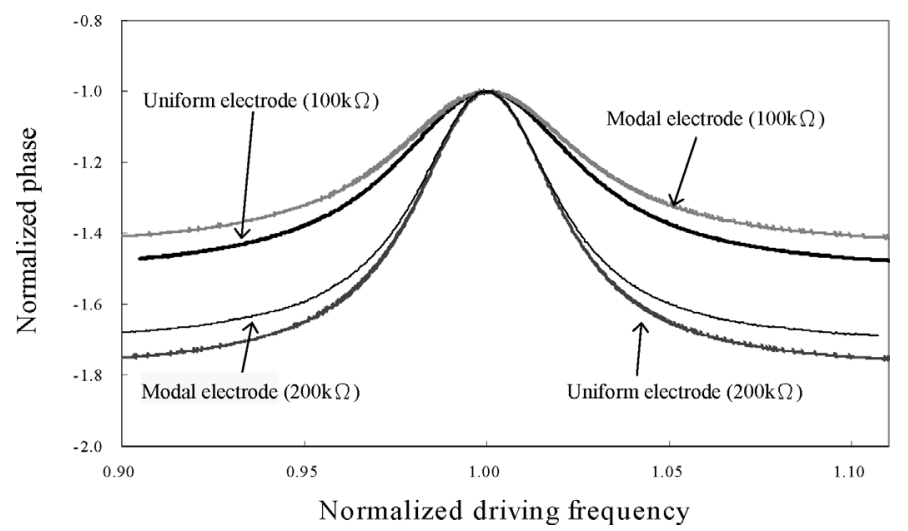

(b)

Fig. 8. Normalized driving frequency results of full-modal and uniform piezoelectric transformers. (a) Magnitude. (b) Phase.

electric transformers, a $0.1 \mathrm{uF}$ capacitance was connected in parallel to the full-modal, piezoelectric transformer in order to compensate for the capacitance difference. With this compensating component in place, a $26 \mathrm{uH}$ inductor was then used to produce the desired LC resonance. Taking the above-mentioned inductance and capacitance into account, the LC resonant frequency of the modal and uniform piezoelectric transformer was calculated to be $75.6 \mathrm{kHz}$ and $71.6 \mathrm{kHz}$, respectively. The two resonant frequencies were found to be very close to each other, and both are slightly higher than the resonant frequency of the piezoelectric transformer. The experimental results are shown in Fig. 11. The data were recorded by using an oscilloscope (LT364, LeCroy Corporation, Chestnut Ridge, NY). The input and output voltage was measured by using 10x and 100x voltage probes (P6109A and P5100, Tektronix Inc.) with an oscilloscope. The input current was measured by using a current probe and a current amplifier (A6302 and AM503B, Tektronix Inc.) together. The output current was measured using a Tektronix AC current probe (CT2, Tektronix Inc.). The input/output power of the piezoelectric transformer then was calculated by multiplying these waveforms together. After the lamp was ignited, the driving frequency was close-looped controlled by using the tube current. The constant current feedback control algorithm served two purposes: to keep the lamp 


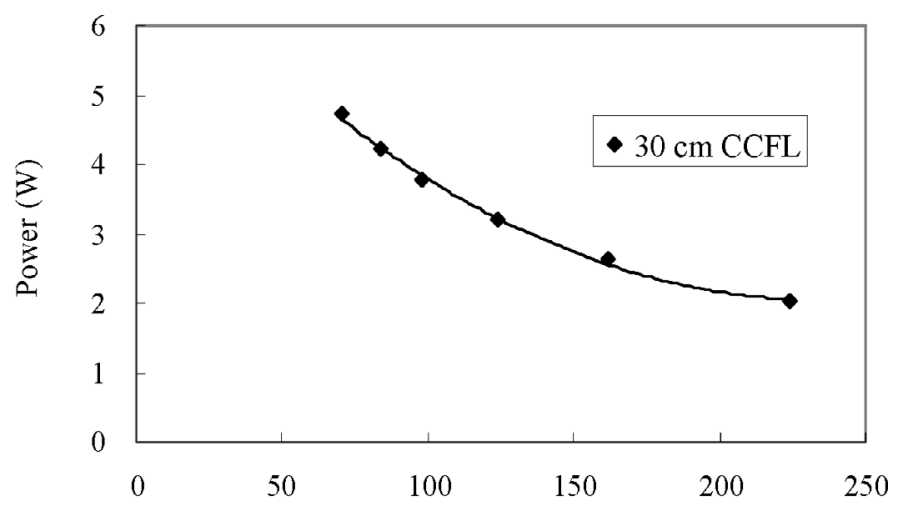

Equivalent impedance $(\mathrm{k} \Omega)$

Fig. 9. Properties of a 300-mm long CCFL.

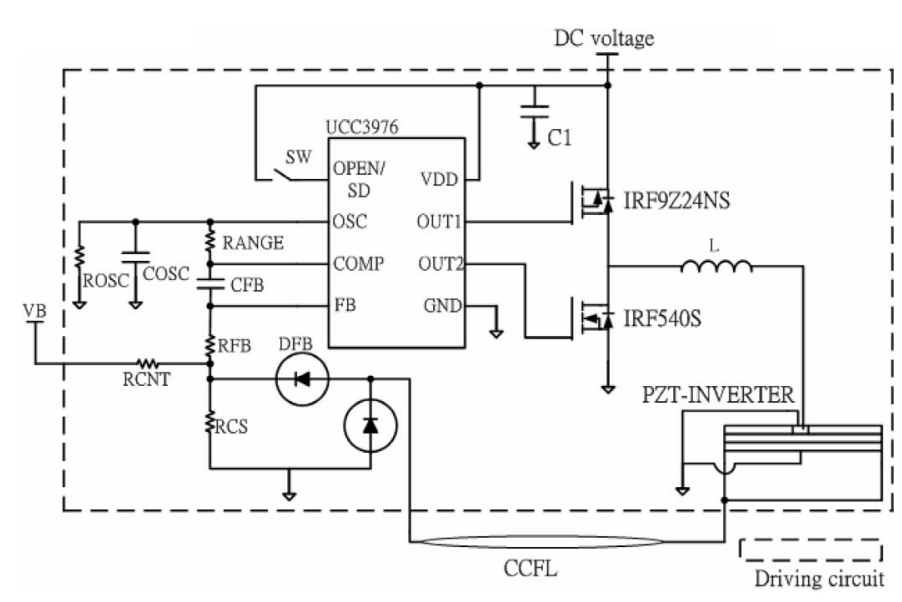

Fig. 10. Driving circuit of a piezoelectric-based inverter.

brightness constant, and to make sure the piezoelectric transformer worked within the optimal working frequency range. It should be noted that this LC resonant tank could filter out some high-order harmonics on the switched frequency, which is typically used for traditional piezoelectric transformers. Nevertheless, some spillover noise still can escape the LC resonance filtering and be discharged. However, this discharged noise can be effectively eliminated by using our newly developed, full-modal electrode. In Fig. 11(a), the working frequency was $65.6 \mathrm{kHz}$, and the input power was $3.32 \mathrm{~W}$ and output power $2.96 \mathrm{~W}$. The energy transfer efficiency was determined to be $89.1 \%$. In Fig. 11(b), the working frequency was $65.8 \mathrm{kHz}$, the input power $3.27 \mathrm{~W}$ and output power $3.05 \mathrm{~W}$. The energy transfer efficiency was determined to be $93.3 \%$. In summary, a full-modal, piezoelectric transformer possesses higher energy efficiency than that of a traditional type due to the effective modal filtering provided by the input electrode. Traditionally, the driving circuit of the piezoelectric transformer uses an inductance to adjust the shape of the input voltage. Nevertheless, it is difficult to choose a perfectly matched inductance for all the operating conditions that a piezoelectric transformer may encounter. By using a fullmodal input electrode, our results show that the piezo-

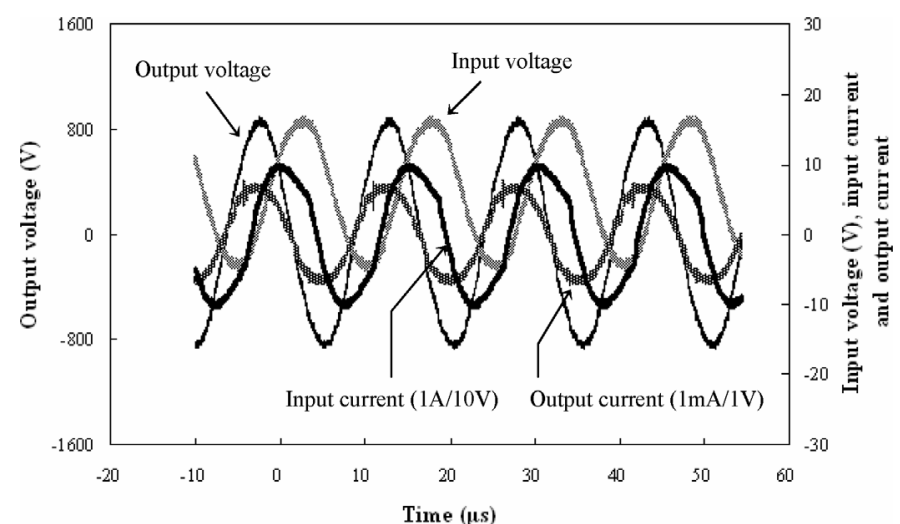

(a)

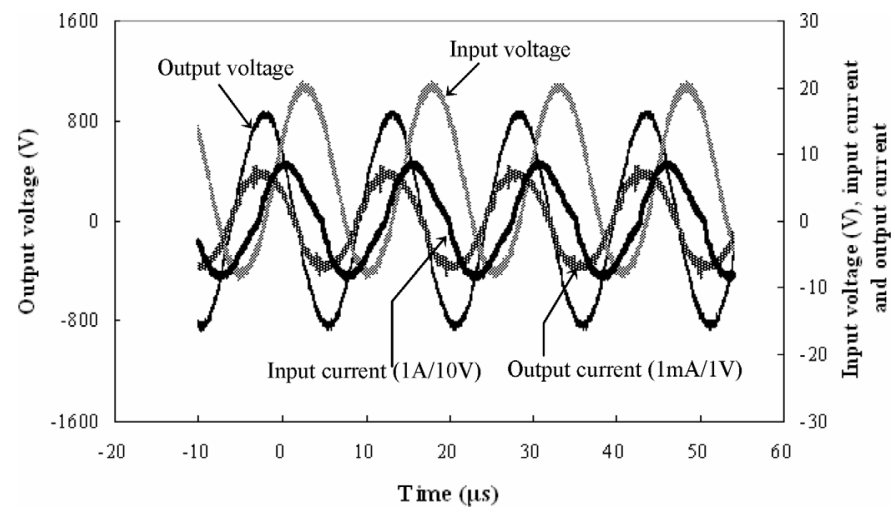

(b)

Fig. 11. Experimental voltage and current waveforms for piezoelectric transformers. (a) Uniform electrode. (b) Full-modal electrode when connected to light a $30-\mathrm{cm}$ CCFL.

electric transformer can achieve higher energy transfer efficiency. In addition, its wider optimal working frequency range makes the driving circuit easier to control.

The full-modal, piezoelectric transformer was used to raise the direct current (DC) voltage to the desired alternating current $(\mathrm{AC})$ voltage. The $\mathrm{DC}$ voltage was derived from the DC power supply, and the voltage was lower than $13.5 \mathrm{~V}$. A 300-mm CCFL was used, and the tube current was sensed by using a control IC (UCC3976, Texas Instruments). The frequency of the input voltage was varied to keep the tube current constant, which in turn allowed the piezoelectric transformer to remain operating in the optimal working frequency range. The maximum energy transfer efficiency of the piezoelectric transformer was found to be about $94 \%$, and the temperature rise was lower than 5 degrees; the CCFL tube current was about $5 \mathrm{~mA}$.

\section{Conclusions}

An innovative, multilayered, piezoelectric transformer, which adopts a full-modal concept in the design of the input electrode shape, was developed. The normalized driving factor $r_{i b}$ was used to theoretically examine the fullmodal filtering effect. The characteristics of a voltage gain transfer function and input impedance were experimen- 
tally measured to demonstrate the modal-filtering effect of the full-modal electrode. The energy transfer efficiency of the full-modal, piezoelectric transformer was found to be higher than that of a distributed type. A wider optimal working frequency range also was found to ease the feedback control algorithm. An inverter, developed by incorporating the newly developed full-modal, piezoelectric transformer, was designed and fabricated to light a 300mm CCFL. The piezoelectric transformer was found to have more than $3.5 \mathrm{~W}$ in output power while possessing $94 \%$ power efficiency. The total temperature rise of the inverter was less than 5 degrees. All these characteristics clearly demonstrate the excellent features and advantages of the newly developed, multilayer, modal actuator-based, piezoelectric transformer that easily can be incorporated into the design of a broad range of potential applications.

\section{ACKNOWLEDGMENTS}

The authors would also like to thank Eleceram Technology Co. Ltd. for continuously providing us with piezoelectric transformers and for some portion of the research funding for this research work. They also would like to thank Texas Instruments (USA) for generously providing us with the control ICs. The continuous financial support from AdvanceWave Technologies, Inc., the Materials Research Laboratory of the Industrial Technology Research Institute (ITRI), and Taiwan National Science Council through NSC 95-2622-E-002-003 is also greatly appreciated.

\section{REFERENCES}

[1] M. Yamamoto, Y. Sasaki, A. Ochi, T. Inoue, and S. Hamamura, "Step-down piezoelectric transformer for AC-DC converters," Jpn. J. Appl. Phys., vol. 40, pp. 3637-3642, May 2001.

[2] M. Yamamoto, Y. Shimada, Y. Sasaki, T. Inoue, K. Nakamura, and S. Ueha, "A multilayered piezoelectric transformer operating in the third order longitudinal mode and its application for an inverter," IEICE Trans. Electron., vol. E85-C, no. 10, pp. 1824-1832, Oct. 2002.

[3] B. Koc, Y. Gao, and K. Uchino, "Design of a circular piezoelectric transformer with crescent-shape input electrodes," Jpn. J. Appl. Phys., vol. 42, pp. 509-514, 2003.

[4] C. K. Lee, "Piezoelectric laminates for torsional and bending modal control: Theory and experiment," Ph.D. dissertation, Cornell University, Ithaca, New York, 1987.

[5] C. K. Lee, "Theory of laminated piezoelectric plates for the design of distributed sensors/actuators: Part I. Governing equations and reciprocal relationships," J. Acoust. Soc. Amer., vol. 87, pp. 1144-1158, Mar. 1990.

[6] C. K. Lee, "Piezoelectric laminates: Theory and experiments for distributed sensors and actuators," in Intelligent Structural Systems. Norwell, MA: Kluwer, 1992, pp. 75-167.

[7] Y. S. Hsu, C. K. Lee, and W. H. Hsiao, "Optimizing piezoelectric transformer for maximum power transfer," Smart Mater. Struct., vol. 12, pp. 373-383, June 2003.

[8] Y. S. Hsu, C. K. Lee, and W. H. Hsiao, "Electric and mechanical fully coupled theory and experimental verification of Rosen-type piezoelectric transformers," IEEE Trans. Ultrason., Ferroelect., Freq. Contr., vol. 52, pp. 1829-1839, Oct. 2005.

[9] Y. T. Huang, W. H. Hsiao, Y. S. Hsu, and C. K. Lee, "Experimental investigation of multi-layer modal actuator-based piezo- electric transformer," in Proc. 15th Int. Conf. Adaptive Struct. Technol. (ICAST 2004), Bar Harbor, ME, Oct. 24-27, 2004.

[10] Y. T. Huang, W. H. Hsiao, Y. S. Hsu, and C. K. Lee, "Highpower backlight inverter for LCD-TV using piezoelectric transformers," in Proc. 16th Int. Conf. Adaptive Struct. Technol. (ICAST 2005), 2005, pp. 376-384.

[11] C. Y. Lin, "Design and analysis of piezoelectric transformer converters," Ph.D. dissertation, Department of Electrical Engineering, Virginia Polytechnic Institute and State University, Petersburg, VA, 1997.

[12] K. Sakurai, K. Ohnishi, and Y. Tomikawa, "Presentation of a new equivalent circuit of a piezoelectric transformer under highpower operation," Jpn. J. Appl. Phys., vol. 38, pp. 5592-5597, Sep. 1999.

[13] J. Shimamura, M. Sakamoto, and K. Kamitani, "Method and apparatus for driving piezoelectric transformer," U.S. Patent No. 6,348,755, Feb. 2002.

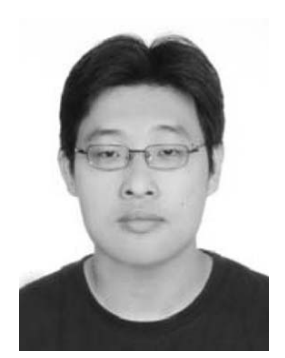

Yao-Tien Huang received his B.S. degree in mechanical engineering in 2001, and a M.S. degree in applied mechanics in 2002, from the National Taiwan University, Taipei, Taiwan. He is currently a Ph.D. candidate at the Institute of Applied Mechanics, National Taiwan University.

His major research interests include the design and applications of piezoelectric transformers.

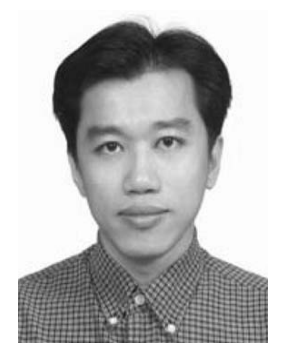

Wen-Jong Wu received his M.S. and Ph.D. degrees from the Institute of Applied Mechanics, National Taiwan University, Taipei, Taiwan, in 1998 and 2003, respectively. Since 2003, he has been an assistant professor at the Department of Engineering Science and Ocean Engineering, National Taiwan University.

His research interests include systems design, integration of precision metrology, smart sensor networks, and piezoelectric power devices.

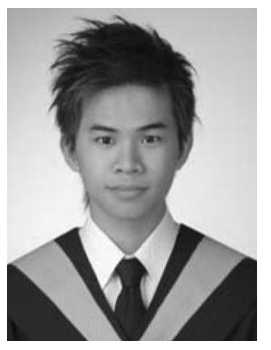

Yen-Chieh Wang received his B.S. degree in mechanical engineering from the National Taiwan University, Taipei, Taiwan, in 2005. $\mathrm{He}$ is currently a M.S. candidate in the Department of Engineering Science and Ocean Engineering, National Taiwan University.

His major research interests include the applications of piezoelectric transformers.

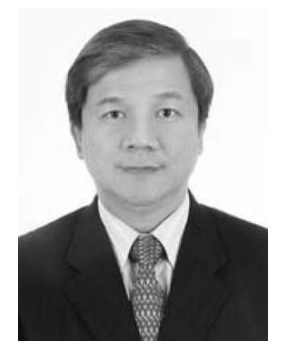

Chih-Kung Lee received his M.S. and Ph.D. degrees in theoretical and applied mechanics from Cornell University, Ithaca, NY. He was with IBM's Almaden Research Center in San Jose, CA, for 7 years. In 1994, he joined the faculty at the Institute of Applied Mechanics, National Taiwan University, Taipei, Taiwan. He is currently holding a 4-year appointment as Director General of Engineering and Applied Sciences at Taiwan's National Science Council, Taipei, Taiwan.

His research interests include microelectromechanical systems, nano systems, piezoelectric systems, automation, optoelectronic system design and fabrication, precision metrology, and biochip systems. 